Diánoia, vol. 15, no. 15, 1969

\title{
LA PREDICCIÓN Y SUS CONSECUENCIAS
}

La predicción de los acontecimientos futuros es el resultado de una inferencia, o de un grupo de inferencias, establecido con base en los conocimientos que se tengan del estado presente y de las leyes que gobiernan el comportamiento de un proceso, o conjunto de procesos, extrapolando esos datos hasta un intervalo de tiempo futuro. Por predicción denotamos tanto el proceso de ejecución de la inferencia, o grupo de inferencias, como la expresión de su resultado. El objeto de la predicción puede ser un acontecimiento que surgirá en el futuro, la repetición de un acontecimiento ya ocurrido en el pasado, o bien, un acontecimiento que ya está sucediendo, pero que todavía no sea conocido. En este último caso, la predicción se refiere a alguna de las manifestaciones futuras del acontecimiento respectivo. El acontecimiento previsto puede consistir en la invariancia del comportamiento del proceso o conjunto de procesos, en una transformación de dicho comportamiento, en la desaparición de un cierto proceso, en la producción de algún proceso nuevo, o bien, en cualquier incidente que se pueda presentar en el desarrollo del proceso o conjunto de procesos en cuestión.

Uno de los postulados primordiales en que se basa la ciencia, tanto en su conjunto como en cada una de sus disciplinas integrantes, es que el desarrollo de los procesos existentes es predictible y verificable, incluyendo los acontecimientos extremos de su surgimiento y su desaparición. En rigor, la predictibilidad y la consiguiente verificabilidad de las predicciones, se encuentran implicadas de manera necesaria en los conceptos, las hipótesis, las leyes, las teorías, los principios, los modelos y, en general, en cualquier explicación científica. Más todavía, la mera consideración de que fuese imposible hacer predicciones, o de que éstas no se pudiesen verificar, equivaldría a negar por completo el conocimiento científico.

El concepto es la sintesis en la cual se expresan los conocimientos adquiridos acerca de un proceso o grupo de procesos, de alguna de sus propiedades o de una de sus relaciones con otros procesos. Asi, el concepto permite comprender mejor los datos conocidos y sirve para descubrir otros aspectos y nuevas relaciones. Muchas veces, tales aspectos y relaciones se encuentran contenidos implícita o explícitamente en la formulación inicial del concepto $y$, en otros casos, resultan de las sucesivas reformulaciones que van enriqueciendo al concepto en el curso de su desarrollo. Pero, siempre, la condición ineludible para establecer racionalmente una nueva conexión conceptual, es la posibilidad de su verificación en la experiencia. Por ende, cualquier am: pliación cuantitativa o cualitativa en el contenido de un concepto, tiene que sugerir posibles efectos experimentales. De tal manera que la predicción de 
esos efectos, que luego deben ser sometidos a la prueba de la verificación, es algo inherente e inseparable del concepto.

Las hipótesis establecidas científicamente, además de ofrecer una explicación suficiente de los hechos a que se refieren, tienen que conducir racionalmente a la predicción teórica de algunos otros acontecimientos. Porque son justamente las consecuencias posibles extraídas de una hipótesis, mediante una o varias predicciones, las que son sometidas después a la prueba del experimento, para poder decidir acerca de la validez de esa hipótesis. Por lo tanto, las hipótesis no sólo deben permitir, sino también facilitar, la ejecución de inferencias que llevan a predecir hechos nuevos, a sugerir nuevas experiencias y a formular nuevas hipótesis. Cuando una hipótesis responde a dichas exigencias, puede servir para orientar el rumbo de las investigaciones subsiguientes, tanto en el terreno de la experiencia como en el dominio del desenvolvimiento racional.

Los cambios y transformaciones que sufren los procesos están regulados por relaciones constantes, a las que denominamos leyes objetivas. Esas regulaciones, cuando son conocidas, se expresan en la forma de proposiciones universales definidas y reciben el nombre de leyes científicas. En muchos casos, las leyes cientificas se pueden enunciar en forma de ecuaciones. Cada ley científica expresa una relación necesaria, que se cumple conforme a ciertas condiciones y cuyos efectos se manifiestan en acontecimientos determinados. Los efectos dependen tanto de las leyes como de las condiciones específicas; de tal manera que el cumplimiento de una misma ley produce resultados diferentes, cuando cambian las condiciones específicas. Por lo tanto $-\mathrm{y}$ éste es un conocimiento fundamental que el hombre tiene desde el comienzo mismo de su actividad científica-, aun cuando no se pueden cambiar las leyes, ni tampoco es posible sustraerse a su cumplimiento, sin embargo, sí es factible cambiar las condiciones especificas y obtener consecuentemente otros efectos.

Las leyes no determinan el comportamiento particular de los procesos, sino que lo regulan en condiciones determinadas. Ninguna ley, por sí sola, puede anticipar lo que ocurrirá singularmente a un cierto proceso; es decir, que no permite predecir algún acontecimiento en particular. Lo que sí puede anticipar una ley es lo que sucederá -esto es, el acontecimiento particular que se producirá- cuando se cumplan tales y cuales condiciones. En este sentido, las leyes científicas desempeñan la función de predecir lo desconocido - como son los cambios que se operarán en un proceso- con base en lo conocido, esto es, las condiciones que se han determinado. Así, cuando una ley ha quedado comprobada, explica los procesos que determinaron su formulación y, a la vez, predice el comportamiento futuro de esos mismos procesos y de todos los otros procesos pertenecientes a la misma clase. De esa manera, la generalización de una relación necesaria, expresada a tra- 
vés de una ley científica, constituye una predicción universal dentro del dominio de su cumplimiento.

Las relaciones constantes que regulan los cambios y transformaciones de los procesos existentes, son constricciones en su comportamiento. Sin tales constricciones, el universo sería completamente caótico. La organización de los procesos, sus ordenaciones, sus simetrias, sus interacciones, sus movimientos, junto con las regularidades de su comportamiento y muchas otras modalidades de su existencia, imponen un gran número de construcciones a los procesos. Cada ley científica es, entonces, la expresión determinada de una constricción. Así, por ejemplo, la ley de Newton sobre el movimiento planetario establece que, entre todas las posiciones y velocidades posibles, solamente un pequeño grupo es el que se cumple en la realidad. En este sentido, la ley excluye muchas posiciones y un enorme número de velocidades de los planetas, prediciendo que nunca se producirán. Por consiguiente, el establecimiento de la ley es una predicción negativa. Por otra parte, la misma constricción inexorable impuesta por la ley científica, aunada al conocimiento de las condiciones especificas, es lo que permite predecir los acontecimientos futuros. En rigor, la posibilidad misma de hacer cualquier predicción implica, ineludiblemente, la existencia de alguna constricción conocida.

Una teoría científica está constituida por un conjunto de leyes ordenadas sistemáticamente, que permite explicar el comportamiento de los procesos en un nivel determinado de la existencia. Cuando una teoría se encuentra suficientemente desarrollada es susceptible, en principio, de ser expresada en la forma de un sistema postulativo, con sus correspondientes axiomas, definiciones, reglas de operación y teoremas. Entonces, siempre que la teoría sea completa y se encuentre bien formulada - dentro de las limitaciones insuperables que las pruebas de Goedel y de Cohen han puesto de manifiesto acerca de la completud y la buena formulación de los sistemas postulativos-, se desprenden de ella tres consecuencias importantes. En primer lugar, las leyes conocidas se pueden inferir de la teoría, en la forma de teoremas y, además, son susceptibles de verificación experimental. En segundo lugar, la teoría explica las leyes que la constituyen, agregando algo más que no está contenido en las leyes, consideradas por separado, sino únicamente en su conjunto. Por último, la teoría predice y explica por anticipado otras leyes nuevas, o sea, que conduce al descubrimiento de regularidades cuya existencia no se sospechaba antes de que la teoría quedara formulada sistemáticamente.

En rigor, la función principal de una teoría cientifica consiste en permitir, partiendo de una determinación o una medición inicial efectuada por un investigador, la inferencia o el cálculo de las predicciones concernientes a los resultados de determinaciones o mediciones ulteriores, ya sea que las ejecute 
el mismo investigador $u$ otros. Una teoría es científicamente válida cuando explica los procesos ya comprobados dentro de su dominio, lo mismo que los otros procesos pertenecientes al mismo nivel de la existencia, aun cuando todavía no hayan sido experimentados. Lo que es más, cuando se impone la necesidad de formular una teoría de mayor amplitud, por haberse descubierto hechos que no pueden ser explicados por medio de las teorías establecidas, entonces es indispensable que la nueva explicación teórica, además de explicar los hechos conocidos, entre los cuales están incluidos los que impusieron la necesidad de hacer esa nueva interpretación, también permita la predicción o anticipación teórica de otros hechos y haga posible su verificación experimental.

Los principios científicos expresan aquellas regularidades en el comportamiento de los procesos, que se cumplen en varios niveles de la existencia o, inclusive, en el universo entero. Por consiguiente, los principios forman parte integrante de varias teorias cientificas, o bien, de todas ellas a la vez. De esa manera, los principios no solamente son elementos constituyentes básicos de las teorías respectivas, sino que permiten diseñar la forma de las leyes -esto es, predecirlas-, hasta en el caso de que los datos experimentales no sean suficientes. En ciertas circunstancias, los principios científicos llegan a servir también para predecir la estructura de las nuevas teorías, cuando se impone la necesidad de establecerlas. En fin, cuando se logra poner de manifiesto el modo específico en que se cumple la regulación expresada por un principio científico en un proceso concreto, entonces es posible obtener mayor información acerca de ese mismo proceso o, por lo menos, es posible predecir con una aproximación muy grande, algunas de sus propiedades que todavía se desconozcan experimentalmente.

Los modelos que se utilizan en la ciencia son representaciones análogas al sistema original que se esté investigando. Por ende, el funcionamiento del modelo tiene que ser equivalente al funcionamiento del original; mientras que los elementos pueden ser completamente diferentes $y$, de hecho, lo son en la inmensa mayoría de los casos. Los modelos pueden ser físicos o dialécticos. Un modelo físico es un mecanismo existente o proyectado, o bien, es un sistema de procesos existentes o hipotéticos. Un modelo dialéctico es un sistema lógico, descrito en un lenguaje preciso - como es, entre otros, el simbolismo matemático- que sea análogo a un sistema de procesos existentes o hipotéticos. En ambos casos, el funcionamiento del modelo, o las operaciones ejecutadas con él, sirve de base para poner de manifiesto nuevas propiedades posibles del sistema original. Los elementos con los cuales se construye o se diseña un modelo físico, pueden ser tomados de diferentes sistemas o adoptados de varias teorías; más todavía, en el modelo se pueden introducir propiedades tecnológicas, las cuales no son consideradas en las teorías. El modelo dialéctico puede ser una teoría ya establecida, pero que corresponda 
a un sistema diferente; pero, también puede servir como modelo una teoría en desarrollo.

Entre el modelo y el sistema original tiene que haber isomorfismo o, por lo menos, el modelo debe constituir un homomorfismo del sistema original. Dos sistemas son isomorfos cuando una transformación biunivoca de los estados de un sistema en los estados del otro, convierte el comportamiento de este último sistema en un comportamiento equivalente al del primero. El isomorfismo es una propiedad recíproca. Además, puede haber isomorfismo entre más de dos sistemas. Por consiguiente, el sistema original es igualmente un modelo del sistema utilizado como modelo. A la vez, un mismo sistema admite como modelos a todos los otros sistemas que sean isomorfos con el primero. Por otra parte, se dice que existe homomorfismo entre dos sistemas, cuando uno de ellos tiene un comportamiento que es equivalente al comportamiento de una versión simplificada del otro sistema. Desde luego, un mismo sistema puede tener varios sistemas homomorfos con respecto a una de sus simplificaciones o con respecto a simplificaciones diferentes. Los sistemas homomorfos son empleados con frecuencia como modelos, debido a que son menos complicados que el sistema original o que los sistemas isomorfos a éste. Más todavía, puede suceder que dos sistemas se encuentren relacionados de tal manera que un homomorfismo de uno de ellos sea isomorfo con respecto a un homomorfismo del otro sistema. En tal caso, uno de estos sistemas también puede servir como modelo del otro. En fin, en muchas ocasiones se pueden utilizar simultáneamente dos o más modelos de un mismo sistema. Entonces, cuando el investigador llega a una parte en que es difícil trabajar con uno de los modelos, puede suceder que la parte correspondiente de otro modelo sea más fácil de entender, de manejar o de investigar.

El funcionamiento del modelo, o la ejecución de operaciones en el modelo, permite advertir propiedades que luego, mediante un razonamiento analógico, son sugeridas como propiedades del sistema original. El razonamiento por analogía, basado en el funcionamiento de un modelo o en el resultado de las operaciones ejecutadas, tiene la ventaja de que permite descubrir propiedades que no figuran todavía en ninguna teoría. Pero, en todo caso, para comprobar la validez del razonamiento, es necesario someter a verificación experimental la existencia de las propiedades sugeridas por la analogía. De esa manera, los modelos se construyen o se adoptan con el propósito de predecir el comportamiento de los procesos integrantes del sistema original. Todo lo que se hace con el modelo son predicciones. En cierto sentido, un sistema sirve como modelo porque permite formular predicciones. Por lo demás, para predecir es necesario establecer modelos, o bien, adoptar como modelos a teorías existentes o mecanismos ya construidos. En general, cualquier concepto, hipótesis, ley, teoría, principio, explicación o, 
inclusive, modelo ya establecido puede servir como modelo o como elemento para configurar un modelo. En todo caso, el cumplimiento de las predicciones formuladas con base en un modelo, es el criterio que permite decidir acerca de la validez de un modelo. Así, cuando la predicción establecida conforme a un modelo no tiene cumplimiento, se hace necesario modificar el modelo. $Y$, en el caso de que se acumulen varias divergencias entre los acontecimientos previstos y los realizados, o de que esas divergencias se repitan con frecuencia, entonces se adopta o construye otro modelo.

La explicación y la predicción científicas son, simplemente, dos aspectos distintos de una y la misma relación lógica. Dicha relación es la que se establece entre los procesos existentes y su determinación por medio del conocimiento científico. La diferencia consiste principalmente en que, en la explicación, la relación se refiere a la determinación de acontecimientos ya realizados, mientras que, en la predicción, la relación se refiere a la determinación por anticipado, o predeterminación, de acontecimientos que todavía no se realizan. La explicación establece las condiciones y leyes, es decir, los explicadores necesarios y suficientes para que se produzca, haya resultado o pueda esperarse que ocurra un acontecimiento, que viene a ser el explicando. Los explicadores son, por una parte, acontecimientos particulares y condiciones especificas $y$, por otra parte, uniformidades o regularidades expresadas por leyes generales. Y el explicando es una consecuencia de los explicadores, considerados en su conjunto. Entonces, cuando conocemos un proceso hasta el punto de haber logrado explicarlo, podemos determinar su comportamiento, tanto en el presente como en el pasado y en el futuro.

En el sentido antes dicho, la predicción consiste en aplicar una explicación determinada a los acontecimientos que se producirán en el futuro. De esa manera, la predicción implica una translación temporal de la explicación establecida, desde un intervalo de tiempo pasado y conocido, hasta otro intervalo de tiempo futuro y por conocer. En todo caso, se considera que, si en ese intervalo de tiempo futuro se cumplen las leyes y se presentan las condiciones que especifican el acontecimiento, entonces dicho acontecimiento ocurrirá conforme a la predicción en cuestión. Como se puede advertir, la posibilidad de efectuar esa translación temporal, sin que se alteren las características de los procesos, ni tampoco el curso de su comportamiento, se apoya en la persistencia de las condiciones y en la invariancia de las leyes ante la translación temporal, como consecuencia de haberse ejecutado una operación de simetría, la cual se basa en la homogeneidad y la invariancia del tiempo. En algunas ocasiones, es posible determinar con precisión el intervalo de tiempo futuro en que ocurrirá el acontecimiento. Pero, en otros casos, el lapso que transcurrirá antes de que se produzca el acontecimiento previsto, queda indefinido cuantitativamente. Sin embargo, en ambos casos se puede 
tener la confianza de que dicho acontecimiento se producirá tal y como ha sido previsto.

El conocimiento elemental de los cambios que sufren los procesos existentes, se adquiere por medio de la observación. Cuando la observación consiste simplemente en registrar los movimientos y transformaciones percibidas directamente por los sentidos, se consigue establecer determinaciones meramente cualitativas. De esa manera es posible formular predicciones que también son puramente cualitativas, a la vez que indefinidas con respecto al momento en que se realizarán. En cambio, el discernimiento de relaciones cuantitativas en el comportamiento de los procesos y la consiguiente aplicación de los procedimientos de contar y de medir, sirve para establecer determinaciones más precisas y permite hacer predicciones definidas con respecto al momento de su realización. Más aún, el desarrollo de la precisión en los resultados de las mediciones y los cálculos, trae como consecuencia la posibilidad de determinar con exactitud las maneras de reproducir ciertas condiciones, para provocar un resultado previsto, o sea, el cumplimiento de una predicción.

Con la intervención del investigador en el comportamiento de los procesos, a través del experimento, provocando la presentación de las condiciones necesarias para perturbar dicho comportamiento de un modo definido, se encuentra implicada la realización de una predicción y su cumplimiento: consecuente. Además, en el experimento se pueden hacer variar las condiciones, dentro de ciertos límites bastante amplios, haciendo posible la repetición de los procesos o su modificación planeada por anticipado. De esa manera, se tiene la posibilidad de variar ampliamente las predicciones, para someter luego su cumplimiento a la prueba experimental. Con el desarrollo de los procedimientos de medición, el mejor control de las condiciones en que se realizan los experimentos, el empleo creciente de las matemáticas y el avance de las técnicas experimentales, que permiten amplificar la percepción sensorial y penetrar en aspectos del comportamiento de los procesos que no se manifiestan aparentemente, se ha logrado afinar mucho las predicciones, incrementando su exactitud y aproximando su cumplimiento cada vez más a la certeza.

En rigor, el experimento consiste en hacer una intervención planeada en el comportamiento de los procesos, dentro de condiciones controladas por el investigador. Por lo tanto, el experimentador tiene que reflexionar, ensayar, tantear, comparar y conjugar muchos elementos, de muy diversas maneras, para predeterminar las condiciones que sean más adecuadas para la realización del objetivo que persigue. Pero, una vez que consigue predeterminar las condiciones y que logra provocar su presentación, entonces el investigador concentra su atención en los resultados, preocupándose por conocer las perturbaciones que hayan alterado el comportamiento del proceso y por regis- 
trar objetivamente su desarrollo, independientemente de las predicciones que haya forjado al principio. Por consiguiente, el experimento está constituido por tres fases principales. La primera consiste en suscitar la presentación de las condiciones objetivas que se han predeterminado. La segunda fase es la de verificar los resultados producidos por el desenvolvimiento del proceso en esas condiciones, independientemente de las predicciones que sirvieron de base para planear el experimento. Y, la tercera, estriba en comparar los resultados obtenidos efectivamente con las predicciones hechas, para comprobar rigurosamente hasta qué punto y de qué manera se han cumplido.

Descubrir es reconocer la existencia de procesos que no se conocían, o de nuevas propiedades en los procesos ya conocidos, o bien, de alguna nueva relación entre procesos considerados como independientes o vinculados solamente de otra manera. Crear es construir una representación anticipada de la realidad por medio de la imaginación racional. Inventar es concebir y resolver problemas nuevos con respecto a procesos, propiedades o relaciones conocidas de una cierta manera. Sin duda, entre el descubrimiento, la creación y la invención hay tantas coincidencias, que muchas veces es difícil discernir en un caso concreto de cuál de esos actos se trata. En todo caso, el descubrimiento siempre va acompañado por la creación de imágenes racionales y suscita la invención de problemas. A su vez, la creación de representaciones racionales es sugerida por los descubrimientos realizados o por los problemas planteados y, entre otras cosas, suscita la concepción de nuevos problemas y conduce a otros descubrimientos. Ahora bien, la invención y la creación son indudablemente dos formas de la predicción, que se llevan a cabo por medio de la imaginación científica, guiada inteligentemente por la razón y apoyada firmemente en los conocimientos comprobados. En cuanto a los descubrimientos, cuando se ha logrado anticipar teóricamente la existencia de nuevos procesos, de propiedades o de relaciones desconocidas, también se trata ciertamente de otra forma de la predicción. Por lo demás, la predicción de la existencia de nuevos procesos, es menos frecuente que la predicción del comportamiento futuro de procesos ya conocidos.

Hacer una predicción significa conocer algo por anticipado, independientemente de los medios por los cuales se llegue a dicho conocimiento. La predicción se basa en la concepción del mundo establecida por la ciencia y se realiza aplicando el método científico. Cuando los conocimientos adquiridos son ordenados sistemáticamente, entonces es posible insertar después los nuevos conocimientos dentro del sistemạ, sin que se altere la ordenación; o bien, en caso de producirse, las alteraciones son tan leves que no afectan al sistema en su conjunto. De esa manera, la ordenación establecida por la ciencia es válida para formular predicciones acerca de los acontecimientos futuros, ya sea mediante interpolaciones o extrapolaciones, por la predeterminación de posibilidades $o$, inclusive, formulando conjeturas. La predic- 
ción requiere el reconocimiento de que los acontecimientos futuros son el resultado o la consecuencia del desarrollo de los acontecimientos presentes. Por lo tanto, la predicción es posible mediante el análisis de las condiciones anteriores, tanto presentes como pasadas, y de las leyes que rigen el comportamiento de los procesos, las tendencias de su desenvolvimiento y los cauces de su evolución. En particular, ese análisis de las leyes es el que permite determinar el carácter, la magnitud, la dirección y el sentido de los cambios que habrán de ocurrir en el futuro, en relación con el presente.

Las leyes científicas determinan el comportamiento de los procesos solamente en condiciones bien definidas y dejando de tomar en cuenta otras muchas condiciones, que son consideradas aleatorias. Por cierto, uno de los descubrimientos fundamentales de una ciencia tan avanzada como la física, ha sido el reconocimiento de la necesidad de especificar con precisión el dominio de las condiciones que son pertinentes para cada clase de procesos. Dentro de una explicación, aquellos elementos que no constituyen las regularidades caracterizadas por las leyes, son denominadas condiciones específicas o, simplemente, condiciones. De esa manera, hasta donde alcanza el dominio de la explicación, las leyes y las condiciones, conjuntamente, especifican el comportamiento de los procesos. Por supuesto, cada vez que es posible y conveniente agregar una nueva especificación, ésta pasa a formar parte integrante de la explicación, siendo considerada como una condición adicional. Ahora bien, las leyes científicas pueden ser causales, funcionales o estadísticas, según sea el carácter con que hayan sido determinadas las regularidades que las propias leyes expresan. Sólo que, independientemente del carácter que tengan las leyes integrantes de una explicación científica, ésta incluye ineludiblemente a la predicción. Pero la predicción tiene sentido únicamente dentro de una clase definida de procesos, respecto a un cierto conjunto de condiciones y con un grado determinado de aproximación.

Como es sabido, las leyes causales expresan una relación entre dos clases de procesos tal que, la presencia o el surgimiento de un acontecimiento en una de esas clases produce, de manera necesaria y suficiente, la aparición de un acontecimiento determinado en la otra clase, el cual viene a ser un efecto del primer acontecimiento. Cuando las leyes pertinentes son causales, entonces la predicción es una consecuencia lógica inmediata de su aplicación a ciertas condiciones específicas. El carácter causal de las leyes hace que las predicciones, o explicaciones en tiempo futuro, queden implicadas biunívocamente por las explicaciones en tiempo presente, con tal que sean suficientemente completas en cuanto a la situación a la que se refieren. Sin embargo, la certidumbre de la predicción no radica solamente en la corrección lógica de la inferencia, sino que debe ser verificada ineludiblemente en la experiencia al transcurrir el lapso predeterminado, para quedar confirmada. En esas condiciones, la predicción causal consiste en ejecutar una inferencia 
para extraer una consecuencia particular, partiendo de las premisas que describen una situación concreta, o bien, que constituyen una representación abstracta de la misma.

Las leyes funcionales solamente determinan la coexistencia de dos clases de procesos, entre los cuales se produce alguna interacción. Por lo tanto, únicamente expresan cómo ocurren los acontecimientos y en qué orden, poniendo al descubierto una relación necesaria entre ellos, que se puede expresar mediante una función matemática: $y=f(x)$. En esas condiciones, la predicción también es inmediata, porque a cada valor de $x$ le corresponde un valor a $y$, o varios valores, según sea la clase de función de que se trate. De esa manera, conocida la ley funcional que vincula dos o más acontecimientos $A$ y $B$ (o bien, $A$ y $B_{1}, B_{2}, \ldots, B_{\mathrm{n}}$ ), entonces se sabe que, si se produce $A$, necesariamente se producirá $B$ (o bien, $B_{1} \circ B_{2} \circ \ldots B_{n}$ ). El carácter funcional de las leyes hace que las predicciones queden implicadas univoca o multívocamente por las explicaciones en tiempo presente, siempre que éstas sean suficientemente completas acerca de la situación a la que se refieren. La implicación será univoca, cuando se anticipe la producción de un solo acontecimiento en el futuro; y será multivoca, en caso de que se pueda producir uno $u$ otro acontecimiento entre varios posibles. No obstante, la certeza de la predicción no radica en la corrección del cálculo de los valores de la función, sino que debe ser sometida a la prueba de la experiencia, para quedar comprobada. En esas condiciones, la predicción funcional consiste en ejecutar un cálculo para encontrar un valor particular, o los diversos valores posibles, partiendo de los valores que se adjudiquen a las variables de la función matemática que representa abstractamente la situación concreta.

Las leyes estadísticas expresan una relación estocástica, que determina el comportamiento medio o promedio de una clase de procesos, considerada en su conjunto. Dicho comportamiento corresponde a la conjugación de una multitud de acciones, las cuales son independientes unas de otras y se muestran impredictibles singularmente. El cálculo de esos valores medios se realiza con arreglo a la teoría de las probabilidades y sus resultados permiten hacer la predicción de ocurrencias futuras de ciertos acontecimientos, no con una certeza completa, pero sí con una probabilidad tan elevada que, en muchos casos, equivale prácticamente a la certeza. El carácter estadístico de las leyes hace que las predicciones queden implicadas multivocamente por las explicaciones en tiempo presente, siempre que el conjunto de referencia sea suficientemente grande para que la aplicación del cálculo de las probabilidades pueda considerarse fidedigna. En general, las predicciones estadísticas se hacen con un margen de incertidumbre y se expresan por medio de una función de probabilidad o de un grupo de funciones de probabilidad. Después, cuando las predicciones son sometidas a la prueba de la experiencia, se verifica alguna de las posibilidades, dentro de la distri- 
bución de probabilidades establecida, convirtiéndose así en certeza para el acontecimiento particular que efectivamente se produce. Pero, en cambio, seguirá subsistiendo cierta incertidumbre acerca de cuál será el acontecimiento que se producirá concretamente en la experiencia siguiente. De esa manera, la predicción estadística consiste en ejecutar un cálculo para encontrar los valores de probabilidad de las diversas alternativas, partiendo de los valores que se adjudiquen a las variables en la función o grupo de funciones de probabilidad, que sirve de medio de expresión a la predicción.

Cuando las leyes causales o funcionales admiten implicaciones univocas y las condiciones se encuentran bien determinadas, entonces es posible hacer predicciones con la mayor exactitud. En cambio, si las implicaciones que se desprenden de las leyes funcionales o estadísticas son multivocas y las condiciones están determinadas de una manera necesaria, entonces el cumplimiento singular de cada una de las alternativas de la predicción resulta aleatorio, por más que se haya conseguido determinar con toda precisión la probabilidad correspondiente a cada alternativa. En rigor, todas las predicciones cientificas son establecidas con cierta probabilidad. Lo que sucede es que algunas predicciones tienen una probabilidad tan cercana a la unidad, que podemos considerarlas como certidumbres, para cualquier propósito científico o práctico; y, por consiguiente, en tales casos le atribuimos un carácter unívoco a la predicción implicada. El grado de conocimiento adquirido sobre el comportamiento de una clase de procesos, nos permite establecer una cierta distribución de probabilidad acerca de los acontecimientos posibles en dicho comportamiento. En muchos casos, como sucede con frecuencia en la física clásica, la amplitud de esa distribución de probabilidad se puede reducir tanto, que resulta despreciable la imprecisión de la predicción. Sin embargo, en otros casos, inclusive dentro de la misma física clásica, la reducción de la distribución de probabilidad tiene límites, que son insalvables de modo transitorio o permanente, con lo cual se hace apreciable la imprecisión de la predicción.

Últimamente, las investigaciones realizadas en el dominio de la cibernética han aportado un nuevo recurso para formular predicciones. Dicho recurso es aplicable a los sistemas relativamente aislados. Un sistema relativamente aislado es aquel que recibe influencia del resto del universo, pero sólo a través de ciertas vías específicas llamadas entradas y que, a la vez, ejerce influencia sobre el resto del universo, pero solamente a través de ciertas vías especificas denominadas salidas. Cuando el estado distinguible presente de cualquiera de las salidas de un sistema relativamente aislado, se encuentra determinado siempre univocamente por los estados distinguibles pasados y presentes de todas las entradas del propio sistema, entonces se trata de un sistema determinado localmente. Pues bien, en algunas ocasiones, resulta que un sistema determinado localmente sólo es observable de una ma- 
nera parcial, o sea, que no es posible obšervar los estados distinguibles presentes en un momento dado en todas sus entradas. En ese caso, el comportamiento del sistema resulta impredictible para el investigador. Pero, entonces se puede utilizar el recurso de considerar que la historia pasada del sistema ejerce su influencia a través de alguna forma de memoria. En ese caso, la adjudicación de memoria al sistema, como parte de la explicación de su comportamiento, es tanto como admitir que dicho sistema no se puede observar integramente. Sin embargo, utilizando ese recurso, el investigador restituye al sistema su cualidad de ser predictible. Por otra parte, a medida que los problemas científicos se hacen más complejos, los cálculos lógicos y matemáticos necesarios para establecer predicciones, resultan más complicados. Pero, afortunadamente, las computadoras se han convertido en auxiliares sumamente eficientes para la ejecución de tales cálculos. Por otro lado, el desarrollo riguroso del razonamiento por analogía y su utilización como método heurístico, permite establecer predicciones con una probabilidad cada vez mayor y sin tener que pasar analíticamente por todas las etapas lógicas.

En el caso de las llamadas partículas elementales de la microfísica, se ha descubierto y se encuentra determinado con precisión el límite de la predictibilidad, que está expresado en el principio de incertidumbre de Heisenberg. Sin embargo, debido a que esa incertidumbre disminuye con el incremento de la masa, las partículas pesadas como los bariones, los nú- cleos y los átomos se encuentran mucho menos afectados por tal limitación; y el comportamiento de las moléculas y otros agregados mayores, es predictible con una incertidumbre muchísimo menor. En lo que respecta a los procesos de mayores dimensiones, cuyos acontecimientos dependen del comportamiento promedio de un gran número de átomos o moléculas, dichos acontecimientos son predictibles con la precisión que se requiere. Desde luego, la predicción de los macroacontecimientos físicos es mucho más pre.cisa que la predicción de los microacontecimientos. Además, la predicción de los acontecimientos físicos concierne primordialmente a las líneas generales de su desarrollo y los resultados principales de su evolución.

Con respecto a las ciencias sociales, la situación es análoga hasta cierto punto. Los microacontecimientos sociales no influyen individualmente en los macroacontecimientos de la sociedad, sino que solamente el promedio resultante de un gran número de microacontecimientos es el que ejerce una influencia importante. La predicción científica no se refiere a los acontecimientos sociales en su detalle minucioso, sino únicamente a las tendencias fundamentales, las líneas generales de su evolución histórica y sus resultados -determinantes o de mayor importancia. En rigor, entre la macrofísica y la -microfísica se tiene la misma relación que existe entre la macrohistoria y la microhistoria, o entre la macroeconomía y la microeconomía. Pero, es in- 
dudable que el nivel alcanzado por el conocimiento en las ciencias naturales, es muy superior al nivel logrado en las ciencias sociales. El conocimiento de las leyes de la naturaleza está apoyado en la realización de experiencias muy numerosas y reiteradas desde mucho tiempo atrás, por lo que los registros del comportamiento de los procesos ofrecen el máximo de precisión. En cambio, en las ciencias sociales sucede lo contrario. Esto influye directa:y decididamente en la cantidad de predicciones científicas que se puede hacer, en las clases de acontecimientos que son predictibles y en la precisión con que se establecen las predicciones. Por eso, las predicciones que se hacen en las ciencias sociales son en corto número, se refieren a unas cuantas clases de acontecimientos y resultan menos precisas. Sin embargo, desde el punto de vista lógico, las predicciones que se pueden formular en el dominio de las ciencias sociales tienen el mismo carácter y pueden llegar a poseer igual grado de determinación que las predicciones que se hacen en las ciencias naturales, siempre que la profundidad, la amplitud y la precisión de los conocimientos que se tengan acerca de las leyes y condiciones en cuestión, sean equivalentes.

En el dominio de los procesos sociales, la acción colectiva de los hombres es capaz de cambiar de manera significativa las condiciones en que se. efectúan, modificando así consecuentemente los acontecimientos ulteriores. El hombre puede alterar igualmente las condiciones en que se producen los procesos naturales; pero los procesos naturales mismos no pueden cambiar deliberadamente sus propias condiciones. Por consiguiente, esa capacidad humana de cambiar las condiciones en que se realizan los procesos sociales, de los cuales forma parte integrante el hombre mismo, constituye un nuevo factor que influye decididamente en el curso de los acontecimientos.'Además, en situaciones críticas, como son las revoluciones sociales, los hombres son capaces de transformar incluso el régimen de la sociedad. En tal caso, junto con el régimen desaparecen también sus leyes específicas, para ser sustituidas por otras leyes sociales diferentes. Esto constituye igualmente otro factor nuevo, que interviene exclusivamente en el curso de los acontecimientos sociales, a diferencia de lo que ocurre en la naturaleza, en donde las leyes correspondientes a los diversos niveles siempre coexisten y mantienen su cumplimiento inexorable, sin que puedan desaparecer jamás mediante la acción humana, ni tampoco por obra de alguna acción de cualquier otro tipo.

En general, independientemente de la amplitud, profundidad y precisión de los conocimientos pertinentes, únicamente resulta predictible un intervalo relativamente corto del tiempo futuro. Más aún, cualquier predicción es siempre parcial y se encuentra restringida a los procesos mejor conocidos. Por otra parte, con frecuencia, la precisión de las predicciones disminuye enormemente al paso y medida en que el futuro, al cual se refieren las predicciones, se aleja del presente. Además, una predicción determinada tiene 
significado solamente dentro de una clase definida de procesos, se establece con respecto a un cierto conjunto de condiciones y tiene un grado de aproximación bien acotado. En muchas ocasiones, la realización de la predicción establecida como consecuencia de las leyes y condiciones pertinentes, es un acontecimiento que ocurre inexorablemente, debido a que se encuentra fuera de nuestro alcance cualquier modificación de las condiciones. Pero, en otros casos, sf resulta posible cambiar las condiciones y, por ende, hacer que también cambie el efecto correspondiente. Pues bien, cuando existe esa posibilidad de modificar las consecuencias y se decide que así suceda, es necesario alterar las condiciones presentes en forma conveniente para producir el efecto deseado. Entonces, para conseguir esa alteración en el acontecimiento previsto, lo que se requiere es impartir al proceso una acción de retroalimentación (feedback) proyectada hacia el futuro o, dicho más precisamente, una acción de prealimentación (el término en inglés sería feedahead) para que se produzca ese cambio deliberado en el comportamiento del proceso.

La prealimentación es un medio eficaz de corregir la acción, con el fin de modificar un acontecimiento futuro, partiendo del conocimiento de la predicción que se haya establecido acerca de ese mismo acontecimiento. A continuación presentamos un ejemplo ilustrativo de la manera como actúa la prealimentación en una actividad humana relativamente simple. Consideremos el caso de un esquiador que desciende a gran velocidad por la ladera de una montaña. El esquiador sabe por experiencia que su equilibrio depende fundamentalmente de que mantenga su cuerpo en posición perpendicular con respecto al suelo. Así, desde el momento en que advierte el menor cambio en la inclinación de la pendiente, el esquiador se prepara para inclinar su cuerpo hacia adelante o hacia atrás, siempre tratando de mantenerse perpendicularmente al suelo. Aparentemente, esa persona ejecuta una acción de retroalimentación. Pero no es así. Para comprobarlo, basta con suponer que el esquiador se desliza con los ojos vendados. En esa condición, los cambios en la inclinación de la pendiente también repercuten en las articulaciones, en los músculos y en las señales que transmite el sistema nervioso del esquiador, inclusive cuando dichos cambios son mínimos. Sin embargo, cuando el esquiador registra como estímulo el desequilibrio que le produce un cambio en la inclinación de la pendiente, ya no tiene tiempo de reaccionar oportunamente, compensándolo por medio de una acción de retroalimentación $\mathrm{y}$, por consiguiente, le es imposible corregir su posición para evitar la caída. En cambio, en condiciones normales, cuando el esquiador utiliza sus ojos, puede observar los cambios de inclinación que vendrán después con anticipación, y no en el momento en que lo van a registrar los estímulos directos en su sistema nervioso motor. Por lo tanto, a la vez que su vista le permite prevenirse ante lo que le espera -aquí se trata literalmente de una pre-visión a través de su vista-, el esquiador tiene oportu- 
nidad de preparar su cuerpo para hacer frente al cambio venturosamente, impartiéndole una acción de prealimentación que lo hace inclinarlo de manera adecuada y en el momento oportuno. De ese modo, mediante una acción de prealimentación, el esquiador modifica de manera anticipada ese factor, evitando así el efecto del desequilibrio, que produciría fatalmente su caida si no recurriera justamente a la acción de prealimentación indicada. Por otra parte, cuando una persona conduce su automóvil por una carretera, constantemente está utilizando la prealimentación para hacer que el vehículo tome las curvas y ascienda o descienda las pendientes en forma adecuada, basándose en las visiones previas que le ofrecen sus ojos. Igualmente, es una acción de prealimentación la que le permite evitar casi siempre los atropellamientos.

Cuando se ha formulado una predicción apoyada en leyes causales, es enteramente factible impartir luego una prealimentación para modificar los acontecimientos previstos, con tal de que existan posibilidades teóricas y prácticas de alterar las condiciones de una manera adecuada. En el caso de que la predicción se haya establecido con fundamento en leyes funcionales, también puede haber la posibilidad de modificar el resultado anticipado. Cuando así se requiera, será necesario alterar los valores de las variables en forma conveniente, para conseguir el cambio consecuente en el valor de la función matemática que representa de manera abstracta la situación concreta y, luego, aplicar esa alteración en la realidad. Por lo tanto, en tal caso se ejerce igualmente una acción de prealimentación, para producir un efecto predeterminado en el comportamiento del proceso en cuestión. En cuanto a las predicciones basadas en leyes estadísticas, la modificación de los valores de probabilidad de los resultados posibles, depende de los valores que se puedan dar a las variables, dentro de los límites de su variación. En consecuencia, también en esos casos es posible impartir una acción de prealimentación, ya sea para aumentar o para disminuir la probabilidad de una o de varias de las alternativas que ofrece el comportamiento del proceso respectivo. En particular, en el transcurso de los acontecimientos sociales intervienen dos factores nuevos, a los cuales ya hicimos referencia anteriormente. Esos factores son el cambio deliberado de las condiciones en que se producen los procesos y, en situaciones críticas, la transformación de las leyes que gobiernan su comportamiento. La existencia de esos dos factores amplía las posibilidades de que las predicciones sirvan de base para influir sobre los acontecimientos previstos, tanto en sentido positivo como negativo. Semejante influencia se ejerce impartiendo a los procesos en cuestión una prealimentación planeada para que se produzcan los cambios consiguientes.

El fundamento de la prealimentación se encuentra en el hecho de que el hombre adapta sus acciones, no sólo con respecto a las necesidades indicadas por las condiciones presentes y por su desarrollo histórico, sino también 
con respecto a las condiciones futuras. La prealimentación consiste en anticipar teóricamente un acontecimiento, utilizando luego dicha predicción para cambiar las condiciones antes de que se produzca el acontecimiento, de tal manera que ocurra una modificación en el comportamiento del proceso en cuestión. De esa manera, con base en la predicción de un efecto, se obra anticipadamente sobre sus factores, para producir un efecto real diferente. El resultado de la prealimentación es una perturbación en el acontecimiento, que puede consistir en su atenuación, su intensificación, su retardamiento, su aceleración o cualquier otro tipo de modificación, incluyendo su desaparición o su sustitución por otro acontecimiento diferente. En algunas ocasiones, el único propósito que se persigue al ejercer una acción de prealimentación, es el de lograr que el acontecimiento se produzca antes o después del momento previsto; lo cual, muchas veces, es suficiente para que el proceso en su conjunto sufra una perturbación considerable. La prealimentación induce el cambio del efecto, mediante la variación de los factores que lo determinan. De esa manera, a través de la prealimentación, los resultados previstos influyen de un modo importante sobre los resultados reales. Por consiguiente, el conocimiento anticipado que se tenga acerca de los acontecimientos futuros, contribuye a conformarlos efectivamente dentro de ciertas modalidades. $\mathrm{Y}$, en particular, dentro del dominio social, la conciencia que se tenga sobre los acontecimientos futuros, coadyuva activamente para que se puedan producir más temprano o más tarde, o bien, para que se alteren notablemente.

La prealimentación desempeña una función muy importante en la preparación de la acción y coadyuva a incrementar la eficacia de su ejecución. Por ende, la prealimentación es parte integrante de la praxiología y de la cibernética. En efecto, como es sabido, la praxiología es la ciencia que estudia la eficacia de la acción. Por su parte, la cibernética ha quedado definida últimamente como el arte de asegurar la eficacia de la acción. Pues bien, como ya lo expresamos antes, un sistema relativamente aislado se encuentra en interacción con su medio y, por lo general, el medio se transforma junto con el sistema. El medio ejerce acciones sobre el sistema, a través de sus entradas $y$, a su vez, el sistema ejerce acciones sobre el medio, a través de sus salidas. En el estudio praxiológico o cibernético de una acción, es necesario definir expresamente el medio sobre el que se ejerce la acción y, también, la meta de la acción, o sea, la transformación que se trata de realizar sobre el medio. Debido a que el hombre concibe sus acciones antes de ejecutarlas, entonces la primera fase es la preparación de la acción. Dicha preparación lleva a la formulación de un programa de acción. Luego viene la segunda fase, que es la decisión de actuar desarrollando el programa formulado. Tal decisión se adopta conforme a distintos criterios específicos, en los cuales se toman en cuenta, entre otras cosas, las propiedades concretas del medio, las características del sistema que sirve como 
agente de ejecución y las posibilidades reales de alcanzar la meta determinada. La tercera fase es la ejecución de la acción. Dicha ejecución se desarrolla siguiendo el orden temporal fijado en el programa.

Al ejecutarse el primer paso establecido en el programa, el medio es modificado y, por lo tanto, se encontrará en un estado nuevo al iniciarse la ejecución del paso siguiente. Con frecuencia ocurre que ese estado no es el que se habia previsto en el programa. En tal caso es necesario modificar los pasos siguientes del programa y, a veces, se requiere complementar el agente de la acción o hasta sustituirlo por otro. Esa adaptación dinámica del programa y de los instrumentos de ejecución con respecto a la situación real del medio, constituye la guía de la acción. En todo caso, se trata de que la acción sea guiada de tal manera que se alcance la meta asignada, es decir, de modo que la acción resulte eficaz. Toda acción se encuentra limitada en el tiempo, tiene un comienzo y un fin. Por consiguiente, cada acción pertenece a un tipo praxiológico y tiene una cibernética propia. El establecimiento del programa también forma parte de la cibernética de la acción considerada. Para poder formular un programa eficaz, es necesario conocer suficientemente el dominio de la acción. Y, para poder guiar la acción, es indispensable tener conocimiento de las modificaciones que va sufriendo el medio sobre el cual se ejerce la acción.

Dentro de la cibernética específica de una acción, se puede recurrir también a la retroalimentación del sistema. En su forma más simple, la retroalimentación directa consiste en acoplar un sistema con otro, de tal manera que una de las salidas del primero sea simultáneamente una de las entradas del segundo y, viceversa, que una de las entradas del primero sea a la vez una de las salidas del segundo. En rigor, los dos sistemas acoplados en retroalimentación constituyen un solo sistema más complejo. También se pueden establecer acoplamientos en retroalimentación indirectos, o sea, por intermedio de otro sistema o de varios sistemas más. Cuando la retroalimentación es negativa, que es lo pertinente en el problema que venimos examinando, entonces su efecto consiste en corregir constantemente cualquier desviación que se produzca en un estado fijado como estado de equilibrio. Dicho estado de equilibrio será, desde luego, el estado del medio previsto en el programa de acción. De esa manera, la retroalimentación negativa modifica convenientemente las condiciones iniciales, inmediatamente después de que se haya producido en el medio un estado que no corresponde al previsto, para afinar justamente el efecto hasta conseguir que el estado real coincida con el previsto. Cuando la retroalimentación es positiva, entonces su efecto consiste en incrementar cualquier desviación que se produzca en el estado de equilibrio. Naturalmente, la retroalimentación positiva también resulta útil, pero en problemas diferentes al que aquí nos ocupa.

La prealimentación también puede ser utilizada dentro de la cibernética 
específica de una acción determinada. Desde luego, a diferencia de lo que sucede en el caso de la retroalimentación, para aplicar la prealimentación no se necesita esperar hasta que se produzca realmente una desviación en la salida del sistema, para corregirla; ya que es suficiente con poder anticipar la desviación, a través de una predicción. En tal caso, se puede recurrir a un acoplamiento en retroalimentación negativa, para que la desviación sea corregida en cuanto se presente. En esas condiciones, la retroalimentación actúa como un dispositivo automático de prealimentación. En otras ocasiones, cuando se ha establecido una predicción acerca del estado o de la secuencia de estados que se producirá, es posible cambiar adecuadamente las condiciones, impartiendo al sistema una acción de prealimentación, de tal manera que se produzcan realmente otros estados diferentes, planeados por anticipado, o que se modifique el orden de su secuencia. En ese caso, durante la segunda fase de la realización de la acción, una vez tomada la decisión de modificar las consecuencias previstas, se reformula el programa de acción de manera pertinente. Y, después, la ejecución de la acción se desarrolla en la forma y de acuerdo con el orden establecido en el programa modificado.

Es fácil advertir que, en cualquier intervención planeada que el hombre realiza en los procesos naturales o sociales, hace uso de la prealimentación. Porque cualquier cambio que se introduzca en las condiciones existentes, con el propósito de cambiar las consecuencias resultantes, constituye propiamente una prealimentación. De la misma manera, cualquier ensayo de esa intervención, como lo son los experimentos, también incluye como parte integrante una acción de prealimentación. En rigor, el empleo de la prealimentación es tan antiguo como la acción humana realizada para cambiar deliberadamente las condiciones naturales, o bien, para gobernar el comportamiento de los procesos existentes. Sin embargo, en este ensayo es en donde se introduce por primera vez, hasta donde sabemos, el concepto de prealimentación de una manera explícita, expresa y definida. Sin duda, el mejoramiento de las predicciones cientificas, de modo que sean cada vez más precisas y tengan mayores alcances, llevará a ampliar el dominio de aplicación de la prealimentación y aumentará su eficacia en forma considerable. En la actualidad, la misma evolución biológica está siendo expresada en la forma de leyes funcionales y estadísticas, que luego son ordenadas sistemáticamente. En esas condiciones de rigor y precisión, las leyes de la evolución biológica admitirán seguramente muchas aplicaciones fructuosas en el dominio de las predicciones y permitirán, más tarde, la utilización estricta y bien controlada de muchas acciones de prealimentación.

Tal como lo hemos expuesto, las predicciones científicas se formulan con apoyo en las hipótesis establecidas, en los datos experimentales ya obtenidos y en las técnicas de verificación disponibles o que sean factibles de 
usarse dentro de un lapso finito. Después, mediante la acción de la prealimentación planeada adecuadamente y aplicada con eficacia, los resultados previstos como posibles sirven de base para introducir cambios en los propios resultados, cuando éstos se producen realmente. La prealimentación puede ser afinada con exactitud en los experimentos, para poder aplicarla luego con segura firmeza en los amplios dominios de la tecnología. De esa manera, a través de la investigación científica se procura conocer primero el desarrollo de los procesos existentes con toda objetividad. Luego se establecen las predicciones pertinentes acerca de su comportamiento futuro, a continuación se puede adoptar la decisión de modificarlo y, en ese caso, se formula el plan para conseguirlo. Finalmente, se interviene deliberadamente en el curso ulterior de los procesos, modificando las condiciones en que iban a realizarse, mediante una acción de prealimentación. Con esa intervención en los procesos, el hombre consigue dominarlos. Y ese dominio sobre el comportamiento futuro de los procesos existentes, conduce justamente al hombre a mejorar las condiciones de su vida, lo cual constituye el objetivo indeclinable y primordial del conocimiento cientifico.

Instituto de InVestigaciones Filosóficas

EuI DE Gortari

Universidad Nacional Autónoma de México 